\title{
Editorial
}

International Archives of
Allergy
Immund

Published online: June 14, 2014 DOI: 10.1159/000363448

\section{Looking for Sensitization Profiles In Different Populations by Recombinant Allergens}

\author{
Riccardo Asero \\ Ambulatorio di Allergologia, Clinica San Carlo, Paderno Dugnano, Italy
}

Molecular biology has completely changed our approach to the diagnosis (and, as a consequence, the management) of allergic disorders. Only a couple of decades ago, diagnosis and treatment relied uniquely on the use of complex and only partially standardized mixtures of both allergenic and nonallergenic proteins called natural allergen extracts; looking back at what was possible then, one gets the impression of another geological era. The advent of an increasing number of recombinant allergen molecules for routine in vitro testing has revealed completely new insights into the way sensitization to different allergen sources occurs and progresses [1]. It has also taught us that many of the tests using extracts have positive results that are actually due to the corecognition of widely cross-reacting pan-allergens $[2,3]$ and has explained us that the clinical expression of many food allergies largely depends on the physical and chemical characteristics of the sensitizing allergens [4]. The results of serological tests based on allergen molecules can change our way of prescribing allergen-specific immunotherapy [5]. However, they also show us that the 'promised land' of tailored immunotherapy is probably infeasible, due to the enormous number of combinations possible in patients sensitized even to one single allergen source [6].

\section{KARGER}

E-Mail karger@karger.com

www.karger.com/iaa
One of the most interesting applications of the molecular diagnosis of allergic diseases is the comparison between populations of allergic individuals living in different geographical areas. Pioneer studies by Scala et al. [7] were carried out on a large number of allergic subjects living mostly in central Italy. They demonstrated a very high rate of IgE reactivity to Cup a 1 (the top scorer with $42.7 \%$ of the cases) and to Pru p 3 (which sensitized about $10 \%$ of the cases). Interestingly, prevalence showed marked variability depending on age.

In an article recently published in International Archives of Allergy and Immunology, Panzner et al. [8] report the results of the detection of IgE to 112 allergenic molecules by ImmunoCAP ISAC in 826 patients, living in the area of Plzen in the Czech Republic, who were sensitized to at least one pollen-derived molecule. Along with some rather predictable findings, such as that grass pollen represents the main seasonal air-borne allergenic source (with Phl p 1 sensitizing $85 \%$ of the subjects) followed by birch pollen (with Bet $\mathrm{v} 1$ sensitizing virtually all sensitized patients), and that these two sources together cause the vast majority of sensitizations, we find some surprises. For instance, a relatively high frequency of sensitization to molecules specific to Cupressaceae, Oleaceae 
and Platanaceae is observed ( $>10 \%$ of the population in all 3 cases) along with a nonnegligible rate of monosensitizations to $\mathrm{Phl} \mathrm{p} \mathrm{4,} \mathrm{which} \mathrm{is} \mathrm{generally} \mathrm{considered} \mathrm{as} \mathrm{a}$ minor allergen among the grass reactors. In view of the sparse distribution of cypresses in central Europe, quite how $14 \%$ of patients became sensitized to Cup a 1 is not clear (from exposure to juniper or Thuja pollen, or travel in the Mediterranean area?). Although Ole e 1-like molecules are present in different plants including grass, goosefoot, plantain and saltwort, it is likely that the high prevalence of sensitization to this allergen (11\%) in this population represented a genuine primary sensitization to Oleaceae. This pattern resembles that in the surroundings of Milan, an area where olive trees are virtually absent [9]. Here, privet pollen is considered to be the possible primary sensitizer; whether this is also the case in the Czech population is unclear. Ash trees represent another possible source of sensitization to Ole 1 . Sensitization to the plane-specific allergen Pla a 2 in the absence of sensitization to the major allergen Pla a 1 remains unexplained. However, one has to consider that Phl p 4 as well as Ole e 1, Pla a 2 and Cup a 1 are native molecules in the ImmunoCAP ISAC assay; as a consequence, test results might be influenced by sensitization to cross-reactive carbohydrate determinants. In effect, Panzner et al. [8] grant that the patients sensitized to natural purified components were more frequently sensitized to MUXF3 than the other patients were. However, the sensitization rate to crossreactive carbohydrate determinants in the whole study population was $6.5 \%$, which is much less than the proportion of subjects sensitized to $\mathrm{Phl} \mathrm{p} \mathrm{4,} \mathrm{Ole} \mathrm{e} \mathrm{1,} \mathrm{Pla} \mathrm{a} 2$ or Cup a 1.

The total prevalence of profilin sensitization (12.4\%) was found to be lower than in southern Europe [10]. Surprisingly enough, Panzner et al. [8] found significant differences in the prevalence of sensitization to individual profilins, including Phl p 12, Bet v 2 and Mer a 1, with Phl p 12 being the least frequent and Mer a 1 being the most frequent. This finding seems to contradict the generally accepted idea that profilins are largely cross-reacting allergens, and that one single representative profilin may be sufficient to detect the sensitization to the whole group [11]. Whether this observation is due to the lower sensitivity of the ISAC immunoassay as opposed to the CAP singleplex assay warrants investigation in future studies. The authors also found a relationship between the sensitization to species-specific molecules and the respective profilins [8]. This underlines that profilin sensitization may originate from a primary sensitization to different allergen sources [12].

Finally, Panzner et al. [8] found a global rate of sensitization to lipid transfer proteins (LTP) of $6.4 \%$. As they do not report on the prevalence of sensitization to Pru $p$ 3 , the peach LTP that unquestionably represents the primary sensitizer to these pan-allergens in the Mediterranean area, we are not in a position to understand from where this sensitization originates. The possibility that LTP hypersensitivity may be secondary to pollen sensitization is still under debate [13]. The mystery of the virtual absence of food allergy to LTP in non-Mediterranean countries, despite the existence of sensitization to LTP in $>5 \%$ of a population of atopic subjects, has not yet been solved.

The study by Panzner et al. [8] is a novel contribution on patterns of sensitization in central Europe, and is a useful adjunct to our current knowledge. The use of a large panel of recombinant allergenic molecules for diagnostic purposes may lead to the detection of unexpected sensitizations to certain allergen sources, which stimulates reflection and reasoning on the clinical relevance of single-allergen proteins. Future studies with the same methodology on other geographical areas will further improve our understanding of sensitization processes.

\section{References}

Sensitization Profiles by Recombinant Allergens
Int Arch Allergy Immunol 2014;164:106-108 DOI: $10.1159 / 000363448$
3 Valenta R, Hayek B, Seiberler S, BugajskaSchretter A, Niederberger V, Twardosz A, Natter S, Vangelista L, Pastore A, Spitzauer S, Kraft D: Calcium-binding allergens: from plant to man. Int Arch Allergy Immunol 1998;117:160-166. ricardi PM: Molecular spreading and predictive value of preclinical IgE response to Phleum pratense in children with hay fever. $\mathrm{J} \mathrm{Al}$ lergy Clin Immunol 2012;130:894-901.

2 Van Ree R, Voitenko V, van LeeuwenWA, Aalberse RC: Profilin is a cross-reacting allergen in pollen and vegetable food. Int Arch Allergy Immunol 1992;98:97-104.
4 Akkerdaas JH, Wensing M, Asero R, Fernandez Rivas M, Knulst AC, Bolhaar S, Hefle SL, Aalberse RC, van Ree R: IgE binding to pepsin-digested food extracts. Int Arch Allergy Immunol 2005;138:203-208. 
5 Stringari G, Tripodi S, Caffarelli C, Dondi A, Asero R, Di Rienzo Businco A, et al: The impact of component-resolved diagnosis on specific immunotherapy prescription in children with hay fever. J Allergy Clin Immunol 2014, in press.

6 Tripodi S, Frediani T, Lucarelli S, Macrì F, Pingitore G, Di Rienzo Businco A, et al: Molecular profiles of IgE to Phleum pratense in children with grass pollen allergy: implications for allergen-specific immunotherapy. J Allergy Clin Immunol 2012;129:834-839.

7 Scala E, Alessandri C, Bernardi ML, Ferrara R, Palazzo P, Pomponi D, Quaratino D, Rasi C, Zaffiro A, Zennaro D, Mari A: Cross-sectional survey on immunoglobulin E reactivity in 23,077 subjects using an allergenic moleculebased microarray detection system. Clin Exp Allergy 2010;40:911-921.
-8 Panzner P, Vachova M, Vitovcova P, Brodska $\mathrm{P}$, Vlas T: A comprehensive analysis of middle-European molecular sensitization profiles to pollen allergens. Int Arch Allergy Immunol 2014; 164:74-82.

9 Asero R: Analysis of hypersensitivity to Oleaceae pollen in an olive-free and ash-free area by commercial pollen extracts and recombinant allergens. Eur Ann Allergy Clin Immunol 2011;43:77-80.

10 Asero R, Jimeno L, Barber D: Preliminary results of a skin prick test-based study of the prevalence and clinical impact of hypersensitivity to pollen panallergens (polcalcin and profilin). J Investig Allergol Clin Immunol 2010;20:35-38.
11 Villalta D, Asero R: Sensitization to the pollen pan-allergen profilin. Is the detection of immunoglobulin E to multiple homologous proteins from different sources clinically useful? J Invest Allergol Clin Immunol 2010;20:591595.

12 Villalta D, Asero R: Analysis of the allergenic profile of patients hypersensitive to pollen pan-allergens living in two distinct areas of Northern Italy. Eur Ann Allergy Clin Immunol 2011;43:54-57.

3 Asero R, Pravettoni V: Anaphylaxis to plant-foods and pollen allergens in patients with lipid transfer protein syndrome. Curr Opin Allergy Clin Immunol 2013;13:379385. 